\title{
Spatial perspective taking: Effects of social, directional, and interactive cues
}

\author{
Peri Gunalp ${ }^{1} \cdot$ Tara Moossaian $^{1} \cdot$ Mary Hegarty $^{1}$
}

Published online: 19 February 2019

(C) The Psychonomic Society, Inc. 2019

\begin{abstract}
Including an avatar in the array in a spatial perspective-taking test improves performance, but it is not clear why. Different aspects of an avatar, including its directional nature, its agency (perceived ability for action), and its interactivity, may all influence perspective-taking performance. Experiment 1 examined how performance was influenced by a social directional cue (an avatar) and an abstract directional cue (an arrow). Participants performed best in the avatar condition and no better in the arrow condition than in a control condition. These results suggest that directionality of the cue alone is not sufficient to facilitate performance on this task. Experiment 2 compared an avatar to a concrete directional cue that was grounded in everyday experience interacting with objects, but was non-agentive (a chair). There was no significant difference between the avatar and the chair conditions, which both outperformed the control condition. Participants in both experiments and all conditions primarily reported utilizing mental simulation strategies that involved imagining themselves in the array of objects. The results suggest that grounding the task in everyday interactions with people or objects facilitates this mental simulation process and more generally enhances performance on perspective-taking tasks.
\end{abstract}

Keywords Perspective taking $\cdot$ Sex differences $\cdot$ Agency $\cdot$ Mental simulation $\cdot$ Spatial ability

\section{Introduction}

Imagine you are helping a friend navigate from the library on your college campus to meet you for lunch. In order to give her correct instructions, you first must imagine standing where your friend is standing, perhaps outside the front of the library, imagine what she would see from that position, and then plan a route for her to take. The crucial initial step of this process, imagining being in the physical position of your friend, is a form of visuospatial perspective taking, which can be defined as the process of imagining what the world looks like from another persons' position (Galinsky, Ku, \& Wang, 2005). Visuospatial perspective taking can also be achieved by imagining a perspective that is not of another individual. For example, in using a map to navigate one can imagine walking through a city and engaging visuospatial perspective taking based on landmarks along the depiction of a path. Here, we

Peri Gunalp

pgunalp@ucsb.edu

1 Department of Psychological and Brain Sciences, University of California, Santa Barbara, CA 93106, USA examine how social (agentive), directional, and interactive cues influence perspective-taking performance.

Psychologists have characterized perspective taking in different ways. One important distinction in the developmental literature is between two levels of visuospatial perspective taking. Level 1 is the understanding of what is in an observer's line of sight. Level 2 perspective taking is the understanding that different observers have different experiences and perspectives of the same object or scene (Flavell, Everett, Croft, \& Flavell, 1981). For example, if there is an object between you and another person, you may see the front of the object while the other person, directly across from you, may see the back of the object. The present paper focuses on Level 2 perspective taking, using a task in which people must make judgments about the locations of objects in an array from different perspectives (and not merely whether specific objects would be visible from different perspectives).

Cognitive psychologists have characterized perspective taking as an effortful spatial transformation that updates a representation of oneself after imagined movements (rotation and translation) of one's body (May, 2004; Presson \& Montello, 1994; Rieser, 1989). For example, Rieser (1989) demonstrated how two components of spatial perspective taking, rotation and translation, are deliberate, effortful processes 
for adults. In this study participants were placed in the center of an array of five objects and were asked to imagine either facing one of the objects (rotation) or standing by one object (translation), and asked to point in the direction of a second object. Angular error increased with the deviation between a person's actual facing direction and the direction that they had to imagine, a result that has been attributed to both the ability to take a different perspective to one's own, and ability to inhibit one's current facing direction (Gardner, Brazier, Edmonds, \& Gronholm, 2013; May, 2004; Rieser, 1989).

Another characterization of perspective taking is as an automatic social process (e.g., Clements-Stephens, Vasiljevic, Murray, \& Shelton 2013; Samson, Apperly, Braithwaite, Andrews, \& Bodley Scott, 2010; Sebanz, Knoblich, \& Prinz, 2003; Shelton, Clements-Stephens, Lam, Pak, \& Murray, 2012; Tarampi, Heydari, \& Hegarty, 2016; Tversky \& Hard, 2009; Zwickel, 2009). For example, Tversky and Hard (2009) examined the effects an actor in a scene might have on a participant's descriptions of the spatial relationships between two objects. In the control condition (no actor in the scene), participants most frequently described the spatial relationship between two objects from their own perspective. However, in conditions that included an actor in the scene, several participants described the scene from the actor's perspective rather than their own.

Tversky and Hard suggested that including an actor in the scene, and drawing attention to the actor's action or agency, tapped into an automatic social sense-making ability that underlies perspective taking. When a person is present in a scene we may implicitly try to make sense of their presence by determining the role they play in the social scope of the scene or environment, or their potential for action (Costantini, Ambrosini, Cardellicchio, \& Sinigaglia, 2013). This social aspect is distinct from other social factors like feelings of inclusion or exclusion, which have also been shown to have an effect on perspective-taking performance (Clinton, Magliano, \& Skowronski, 2018). This attempt at social sense-making may even precede our more explicit goals to describe the scene (Tversky \& Hard, 2009). However, it is not clear what it is about the actor in this scene that triggers this sense making; that the actor is human (has agency), that he defines a clear orientation, or that his presence makes it easier to mentally simulate being in the display. If an object were added to the scene that was not perceived to have agency (perhaps a non-human object such as a chair, or a symbol such as an arrow) would we still observe enhanced performance?

In related research, Shelton, Clements-Stephens, Lam, Pak, and Murray (2012) examined how the agency of a cue influenced accuracy and response time on a modified version of the three-mountain task (Piaget \& Inhelder, 1967). In this task, participants were shown an image of three buildings and had to indicate which cue around a matching display of three buildings would have that view. Each cue had what Shelton and colleagues called a different degree of agency: one was a wooden, manipulable, human figure artist model (high agency), one was a camera (moderate agency), and another was a triangular block stacked on top of a pedestal (low agency). For each condition, participants saw displays with only one type of cue around the buildings. Social skills (measured via the Autism Quotient; Baron-Cohen, Wheelwright, Skinner, Martin, \& Clubley, 2001) were significantly negatively correlated with accuracy on the task in the artist model condition, such that participants with lower scores on the social subset of the Autism Quotient (indicating better social skills) were more accurate on the perspective-taking task. However, social skills were not significantly correlated with accuracy in the camera or triangle block conditions. These results suggest that perspective taking with a social agent in the scene may not rely on the same cognitive processes as perspective taking when no social agent is present.

In another series of experiments, Clements-Stephens, Vasiljevic, Murray, and Shelton (2013) examined performance on a similar perspective-taking task but with plain stacked block figures (in some conditions called "aliens"), stacked block figures with eyes, wooden manipulable artist models, and Barbie ${ }^{\mathrm{TM}}$ dolls. Participants had higher accuracy with block figures that had eyes than with plain block figures, higher accuracy with plain block figures called "aliens" than plain block figures with no name, and higher accuracy with Barbie $^{\mathrm{TM}}$ dolls than artist models. That is, degree of agency, ascribed by either conceptual or visual features, increased perspective-taking accuracy.

Here, we study the effects of different stimulus properties on individual differences in perspective taking in the Spatial Orientation Test (SOT). The SOT, originally developed by Kozhevnikov and Hegarty (2001) and later refined by Hegarty and Waller (2004), measures perspective-taking ability. Participants are shown an array of objects and are asked to imagine standing at one object in the array, facing a second, and pointing to a third (see the example item in Fig. 1). The score on the test is the average pointing error across trials. In correlational studies, this test was dissociated from measures of mental rotation (e.g., Hegarty \& Waller, 2004). Moreover, in open-ended strategy reports, a majority of individuals report using a strategy that entails imagining themselves in the array rather than imagining the array transforming or rotating, which would be more akin to strategies used in a typical mental rotation task (Kozhevnikov \& Hegarty, 2001). The SOT has been found to be predictive of several measures of navigation performance including learning the layout of new environments and pointing to locations in known environments (Allen, Kirasic, Dobson, Long, \& Beck, 1996; Fields \& Shelton, 2006; Kozhevnikov, Motes, Rasch, \& Blajenkova. 2006; Schinazi, Nardi, Newcombe, Shipley, \& Epstein, 2013; Weisberg, Schinazi, Newcombe, Shipley, \& Epstein, 2014). Tarampi, Heydari, and Hegarty (2016) examined how 

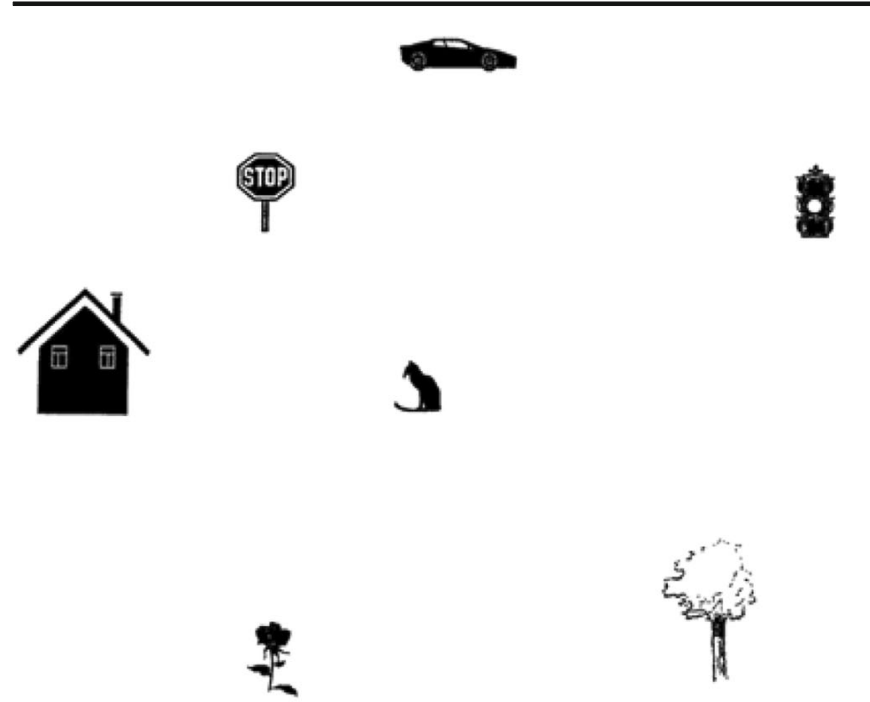

12. Imagine you are standing at the cat and facing the house. Point to the traffic light

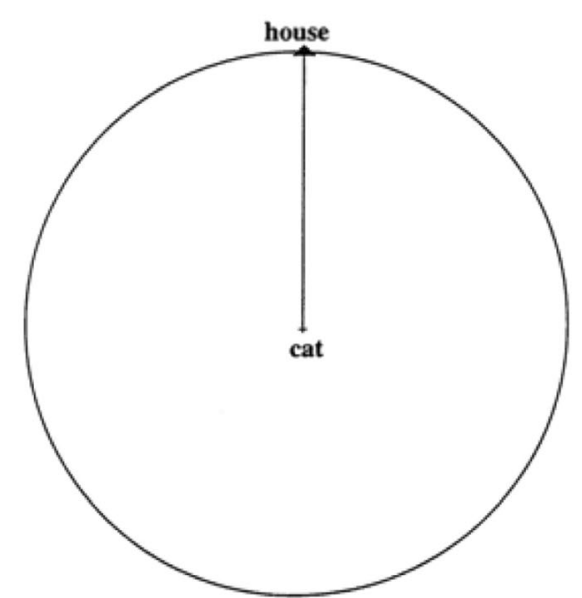

Fig. 1 A sample trial from the control condition of the Spatial Orientation Test (Hegarty \& Waller, 2004)

incorporating a human figure in the SOT influenced sex differences on the SOT. Typically, men have lower pointing error than women on this task. However, Tarampi et al. found that when they introduced a human figure that faced the direction participants were to imagine facing, and asked people to take the perspective of the human figure, women and men performed equally well (Tarampi et al., 2016). Women's performance was better with the inclusion of the human figure in the array, whereas men's performance was equally good in both conditions.

In summary, adding a human figure to a perspective-taking array influences the propensity to adopt another person's perspective (Tversky \& Hard, 2009), the correlation of performance with self-reported social skills (Shelton et al., 2012), and perspective-taking accuracy (Clements-Stephens et al., 2013; Tarampi, Heydari, \& Hegarty, 2016). However, one question that can be raised in response to each of these studies is how the directionality of the agents used may also influence perspective-taking performance. In each of these studies the agents faced in a clear direction within the task display. For instance, in the task used by Tarampi et al., the human figure in the array faced the direction participants were to imagine facing on each trial. In this case the human figure could have made the task easier because it was engaging automatic social processes, or because it provided a clear directional cue.

Additionally, cues with agency may facilitate an enriched mental simulation of imagining oneself in the array. Most mental simulation and grounded cognition theories include the connection of cognition to the human body (e.g., Barsalou, 2008) and to everyday experience. We frequently interact with other people, and therefore have everyday experience of imaging people's perspectives. An arrow on the other hand is not a common object that humans interact with. Although we often see arrows and rely on them as symbols indicating direction, we do not have everyday experience of interacting with arrows. Thus, it might be more difficult to mentally simulate the perspective of an arrow, even though it is a strong directional cue. Moreover, mental simulation may also be facilitated by cues that we interact with in everyday life but do not have agency. For example, one can mentally simulate the action of sitting in a chair, which in turn enables you to imagine the perspective you would have when sitting in a chair.

The main aim of the present paper was to examine possible explanations for previously found effects of agency on perspective-taking performance in the SOT. In Experiment 1, we examine effects of agency and directionality. We measure performance on a new SOT with either a human avatar or an arrow included in the array. In the avatar condition participants are asked to imagine standing at an avatar; this is a directional cue (because it has a clear front and back), and it also has agency. In the arrow condition participants are asked to imagine standing at an arrow, a cue that is directional but abstract (because it is symbolic), and does not have agency. In Experiment 2, we aimed to further distinguish effects of agency, and potential for interaction by comparing an avatar to a chair, that is, a concrete object that we frequently interact with but that does not have agency. Thus, it should be relatively easy to mentally simulate assuming the perspective one would have when sitting in the chair.

In addition to this empirical aim, the present experiments generalize research on adult perspective-taking ability to a new type of display. Previously, measures of perspectivetaking performance in the adult spatial abilities' literature have utilized relatively abstract tasks, such as the Money Road Map task (Money, Alexander, \& Walker, 1965) or the SOT, both of which show a top-down (map like) representation of an environment or array (Hegarty \& Waller, 2004; Kozhevnikov \& Hegarty, 2001; Kozhevnikov, Motes, Rasch, \& Blajenkova, 
2006). In contrast, many experimental studies of perspectivetaking (e.g., Clements-Stephens et al., 2013; Shelton et al., 2012; Tversky \& Hard, 2009) and studies of children's perspective-taking ability (e.g., Epley, Morewedge, \& Keysar, 2004; Huttenlocher \& Presson, 1973, 1979; Newcombe \& Frick, 2010; Newcombe, 1989; Surtees, Apperly, \& Samson, 2013) show views of a real-world scene. The experiments reported here utilize a new virtual reality format for the SOT to explore adult perspective-taking performance in an immersive environment that incorporates naturalistic elements and is more like a real-world scene. In this format, participants view the objects from an oblique perspective (see Fig. 2).

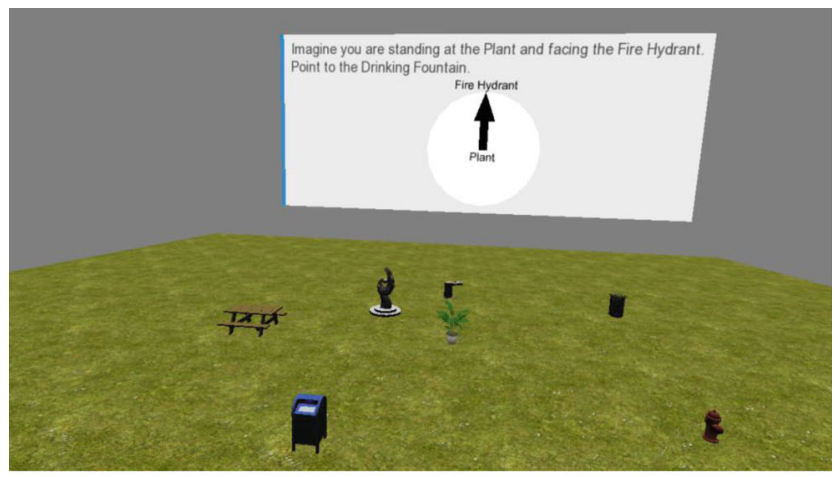

a Control condition.

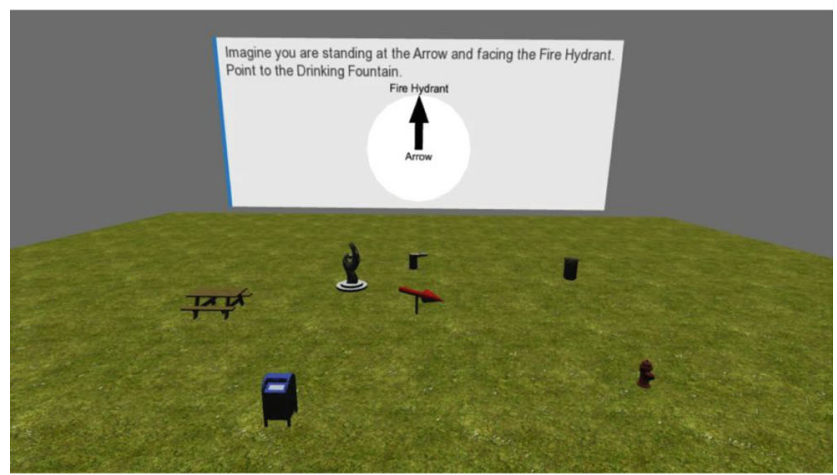

b Arrow condition.

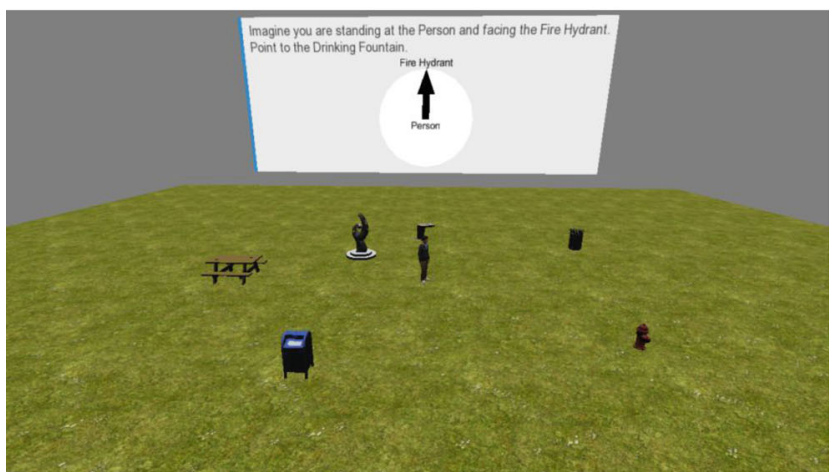

C Avatar condition.

Fig. 2 Screenshots of the virtual reality Spatial Orientation Test from the control (a), arrow (b), and avatar (c) conditions in Experiment 1
To ensure that the new virtual reality (VR) task we used was measuring similar perspective-taking abilities to previous studies, participants completed the paper SOT (Hegarty \& Waller, 2004) at the end of the main experiment. We expected to observe correlations between performance on the main VR and the paper SOTs. Task order was not manipulated because we did not want strategies developed during the paper SOT to affect performance on our main task.

Finally, we used strategy questionnaires to examine the degree to which participants used mental simulation or more abstract strategies in different versions of the perspectivetaking task. The strategy questionnaire was based on previous research with the SOT (Kozhevnikov \& Hegarty, 2001), in which participants responded to open-ended questions about their strategies. Although mental simulation processes are often considered to be automatic and spontaneous (e.g., Niedenthal, Barsalou, Winkielman, Krauth-Gruber, \& Ric, 2005), mental simulation can also be viewed as a strategy in spatial reasoning or learning (e.g., De Freitas \& Sinclair, 2012; Hegarty, 1992, 2004; Nunez, Edwards, \& Matos, 1999). Here, we examine whether different directional cues affect choice of strategy in perspective-taking tasks or whether they merely affect the ease with which participants use a common strategy.

\section{Experiment 1}

In Experiment 1 participants completed the SOT in an immersive VR environment, either in a control condition, with only objects in the array and the perspective to be imagined defined by these objects, an arrow condition, in which the arrow defined the perspective to be imagined, or an avatar condition, in which a human avatar defined the perspective to be imagined. If cue agency is important for improving perspective-taking performance because of an automatic social sense-making process, participants should perform better in the avatar condition than in either the arrow or the control conditions (e.g., Clements-Stephens et al., 2013). However, if the avatar in the array improves performance simply because it provides an additional directional cue, participants should perform better in both the avatar and arrow conditions than in the control condition. Based on the results of Tarampi et al. (2016), we predicted that women would perform as well as men in the avatar condition but poorer than men in the control condition.

\section{Method}

Participants One hundred and thirty-five undergraduate students from the University of California, Santa Barbara participated in this study for course credit. One participant was excluded because they declined to report their sex, and 14 participants (11\%) were excluded for performance at or above chance levels of error (numerically above the chance 
performance score of $90^{\circ}$ ), three (two female, one male) from the control condition, four (two female, two male), from the arrow condition, and seven (five female, two male) from the avatar condition. ${ }^{1}$ Therefore, our final sample had 120 participants, age $17-33(M=19.30, S D=1.79)$ years. A priori power analyses indicated that to detect a medium effect $\left(\eta_{p}{ }^{2}\right.$ $=.13$ ) of directional cue on VR SOT performance at a power of .95 , the necessary sample size would be $n=111$. The present study exceeds this requirement, and therefore is adequately powered to detect this effect.

Design This study had a two-factor between-subjects design. The independent variables were array condition (avatar, arrow, and control), and sex (female and male). There were 59 males (19 avatar, 20 arrow, 20 control) and 61 females (21 avatar, 20 arrow, 20 control) in the final sample. The dependent variable measured was angular error and was averaged across trials within a version of the SOT.

Materials Participants used an Oculus Rift DK2 headset during the Virtual Reality Spatial Orientation Test (VR SOT) trials, which showed a virtual environment that was programmed using Vizard software (WorldViz, Santa Barbara, CA). The single work station consisted of two monitors used for the VR SOT, which were 24-in. Dell P24124 monitors (60-Hz refresh rate) with Nvidia GeForce GTX (660). An Xbox controller was used in the VR SOT trials for participants to submit their answers. None of the Oculus controls were made available to participants.

The virtual environment arrays always contained seven objects. The environment was made to include naturalistic elements and resemble a park scene (see Fig. 2). The ground of the environment was green, and the objects included in the array all could have been found in a park. All trials in the virtual environment were the same format as the trials in the original paper SOT, reading, "Imagine you are standing at $\underline{X}$ facing $\underline{Y}$, point to $\underline{Z}$," where $\mathrm{X}, \mathrm{Y}$, and $\mathrm{Z}$ are all objects in the array. Thus, a trial in the control condition, which mimicked the original SOT, read, "Imagine you are standing at the plant facing the fire hydrant, point to the drinking fountain," (see Fig. 2a). On each trial of the control condition, participants were asked to imagine standing at a different object in the array. Note that the control condition likely has a higher working-memory load than either the arrow or avatar conditions, as in the latter conditions participants imagine standing at the same object on every trial, and that object faces in the direction participants are to imagine facing.

The arrow condition had an arrow that acted as a nonhuman directional cue and replaced one of the objects in the

\footnotetext{
${ }^{1}$ The data were bi-modal, with the second (smaller) mode of participants with average error above chance (raw average error of $90^{\circ}$ ) and more than 2 SDs from the group mean. The analyses described in the text below exclude participants whose average error raw score exceeded $90^{\circ}$.
}

array for each of the trials (see Fig. 2b). A trial in the arrow condition read, "Imagine you are standing at the arrow facing the fire hydrant, point to the drinking fountain." For every trial participants imagined standing at the arrow - the arrow moved accordingly to a new location on each trial (the position of "object $\mathrm{x}$ " in the corresponding control trial), and faced the direction participants were to imagine facing.

The avatar condition had a human male avatar that replaced one object in the array for each of the trials (see Fig. 2c). A trial in the avatar condition read, "Imagine you are standing at the person facing the fire hydrant, point to the drinking fountain." As in the arrow condition, the human avatar was in the same location and faced the direction participants were to imagine facing on each trial. The avatar was not gender matched to participants.

In all conditions the 12 trials were presented in a different randomized order to each participant. On each trial an answer circle was provided perpendicular to/above the array that had a response arrow pre-drawn in it. The response arrow was labeled with both the object by which participants were asked to imagine standing and the object toward which they would be facing. The participant's task was to move the response arrow in the answer circle using the Xbox controller joystick indicating where they would point to the third target object.

Scoring for this task was based on the absolute value of the difference between the angle of the response arrow on the answer circle submitted by the participant and the correct angle (absolute angular error). The correct angle was drawn/ calculated from the center coordinates of each of the three relevant objects; in the arrow and avatar conditions, the center coordinates of the arrow/avatar matched those of the object that was replaced.

On each trial the magnitude of difference between a participant's estimate and the correct answer was determined to yield an angular error score for each trial, and their score on the test was the average angular error across trials. Incomplete trials were assigned an angular error of $90^{\circ}$ (chance performance). Angular error on any trial can range from $0^{\circ}$ to $180^{\circ}$ (from perfect accuracy to pointing in the opposite direction of the correct answer), so if a participant responds by guessing randomly for many test trials, theoretically that participant would have an average angular error of $90^{\circ}$ across items (Sholl, Acacio, Makar, \& Leon, 2000). This procedure is consistent with how incomplete trials were treated in previous research on this task.

Participants were also administered the paper Spatial Orientation Test (SOT) (Hegarty \& Waller, 2004). The paper SOT always showed the same array of seven objects from an aerial view $\left(180^{\circ}\right.$ above the array) and showed only the original (control) array (no human figure or arrow). Below the array there was an answer circle in the same format as the VR SOT on which participants could draw their answer line (see Fig. 1). The order of trials was fixed across participants. 
As in the VR SOT, participants were allowed $5 \mathrm{~min}$ to complete the 12 items of this test and their score was the average angular error across trials.

The Qualtrics online survey platform was used for the questionnaire that contained questions regarding mental strategy used in both the VR and paper SOTs. In a multiple-choice question (see Appendix) participants read four descriptions of different possible strategies identified in previous research on this task (Kozhevnikov \& Hegarty, 2001) and were asked to indicate which strategy was closest to the one that they used for each of the tasks. There was a text-entry option available for participants who felt their strategy was not represented by one of the four multiple-choice options.

Procedure Upon arrival participants gave informed consent and received instructions for the VR SOT by first completing two controller practice trials. Participants were first familiarized with the joystick controls in the context of a simple virtual environment. Next, participants completed two practice trials in which they used the joystick to align the arrow in the arrow circle with a triangle outside the circle. These controller practice trials were completed without the Oculus headset on. Next the headset was put on and adjusted to fit comfortably, and participants completed four practice trials with a four-item array before beginning the test trials.

Before beginning the test trials, participants were asked to memorize and report the names of items in the test array. The participants were then told that they would have $5 \mathrm{~min}$ to complete the trials, the experimenter advanced the screen to the first test trial, and left the room.

After completing the VR SOT trials, participants removed the headset and received the paper SOT packet and read the instructions, after which they were given $5 \mathrm{~min}$ to complete the 12 trials. After $5 \mathrm{~min}$ had passed, or the participant indicated that $\mathrm{s}(\mathrm{he})$ was finished, the experimenter took the paper SOT packet and administered another test, which will not be discussed here. ${ }^{2}$ The experimenter then opened the survey on the computer and told the participant that they had as much time as needed to answer the questions. After completing the survey participants were debriefed.

\section{Results}

A small number of trials were not completed ${ }^{3}$ for the VR SOT $(M=0.16, S D=.57)$ and for the paper SOT $(M=1.01, S D=$ 1.77) and were assigned $90^{\circ}$ (chance performance). As the data for both the VR and Paper SOT tasks were positively skewed, these data were natural-log transformed, and

\footnotetext{
$\overline{2}$ The Money Road map test was used, which is another test of perspective taking, and had moderate correlations with the VR and paper versions of the SOT.

${ }^{3}$ The pattern and significance of the results do not change if we base the analysis on the average error for completed trials only.
}

subsequent analyses are conducted on only log-transformed data. ${ }^{4}$ Table 1 shows descriptive statistics for the raw data.

Virtual-reality Spatial Orientation Test If the avatar activates a social mechanism, participants should perform significantly better in the avatar condition than in both the arrow and the control conditions. If the additional directional cue offered by either the arrow or the avatar facilitates performance, participants should perform better in both the arrow and the avatar conditions than in the control condition.

A 3 (condition: control, arrow, avatar) $\times 2$ (sex: male, female) ANOVA on the VR SOT angular error means revealed a significant main effect of directional cue condition, $F(2,114)=$ $5.55, p=.005, \eta_{p}{ }^{2}=.09$. Post hoc (LSD) pairwise comparisons, corrected for multiple comparisons, indicated that participants performed significantly better in the avatar condition $(M=2.36, S D=0.50)$ than in either the arrow $(M=2.62, S D=$ $0.50, p=.013)$ or the control conditions $(M=2.69, S D=0.47$, $p=.002)$, but there was no significant difference between the arrow and control conditions, $p=.52$ (see Fig. 3). Bayes Factors $\left(\mathrm{BF}_{10}\right)$ for comparison of the avatar condition with the arrow and control conditions were 2.60 and 3.53, respectively, indicating anecdotal evidence for the alternative hypothesis, while the $\mathrm{BF}_{10}$ for the comparison of the arrow and control conditions was equal to .25 , indicating anecdotal evidence for the null hypotheses (all Bayes Factors were computed on Ln error with default priors using JASP 0.8.1.2 [JASP Team, 2017]). These results suggest that directionality alone is not enough to boost performance on this task.

There was also a significant main effect of sex, $F(1,114)=$ $8.13, p=.005, \eta_{p}^{2}=.07$, such that $\operatorname{men}(M=2.44, S D=0.51)$ performed better than women $(M=2.69 S D=0.48)$ overall, $d$ $=.49$ (see Fig. 3 and Table 1). There was no significant interaction between sex and condition, $p=.76$. Analysis of response-time data revealed no significant effect of condition $F(2,104)=.46, p=.63$ or $\operatorname{sex} F(1,105)=2.36, p=.13$, and no interaction, $p=.40$.

Paper Spatial Orientation Test A 2 (sex) $\times 3$ (condition of VR SOT) ANOVA found a significant main effect of sex, $F(1,114)$ $=10.06, p=.002, \eta_{p}{ }^{2}=.08$, such that males $(M=2.48, S D=$ $0.49)$ performed better than females $(M=2.82, S D=0.64), d=$ .59 (see Table 1), replicating previous research on this task (Tarampi et al., 2016). There was no main effect of condition, $F(2,114)=.39, p=.68$, which is to be expected as all participants completed the original version of the paper SOT, so an effect of condition would reflect a difference in paper SOT scores only if it was influenced by which VR directional cue condition the participant was in. There was no significant interaction between sex and condition, $F(2,114)=.49, p=.61$.

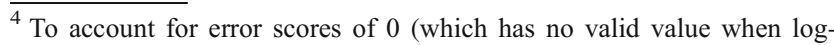
transformed) values of $0^{\circ}$ error were changed to $1^{\circ}$.
} 
Table 1 Experiments 1 and 2: Mean raw angular error for men and women in the control, arrow, chair, and avatar virtual reality (VR) Spatial Orientation Test (SOT) conditions (Experiment 1, $n=120$; Experiment 2, $n=185$ )

\begin{tabular}{|c|c|c|c|c|c|}
\hline & & \multicolumn{2}{|c|}{ VR SOT } & \multicolumn{2}{|c|}{ Paper SOT } \\
\hline & & $\begin{array}{l}\text { Males } \\
M(S D)\end{array}$ & $\begin{array}{l}\text { Females } \\
M(S D)\end{array}$ & $\begin{array}{l}\text { Males } \\
M(S D)\end{array}$ & $\begin{array}{l}\text { Females } \\
M(S D)\end{array}$ \\
\hline \multirow[t]{3}{*}{ Experiment 1} & Control & $\begin{array}{l}24.66 \\
(12.37)\end{array}$ & $\begin{array}{l}27.81 \\
(11.59)\end{array}$ & $\begin{array}{l}21.83 \\
(12.87)\end{array}$ & $\begin{array}{l}2.75 \\
(0.65)\end{array}$ \\
\hline & Arrow & $\begin{array}{l}23.45 \\
(17.88)\end{array}$ & $\begin{array}{l}30.22 \\
(18.14)\end{array}$ & $\begin{array}{l}20.04 \\
(9.95)\end{array}$ & $\begin{array}{l}2.92 \\
(0.64)\end{array}$ \\
\hline & Avatar & $\begin{array}{l}15.51 \\
(7.53)\end{array}$ & $\begin{array}{l}21.7 \\
(13.09)\end{array}$ & $\begin{array}{l}24.88 \\
(18.55)\end{array}$ & $\begin{array}{l}2.68 \\
(0.57)\end{array}$ \\
\hline \multirow[t]{3}{*}{ Experiment 2} & Control & $\begin{array}{l}30.42 \\
(19.53)\end{array}$ & $\begin{array}{l}31.73 \\
(17.41)\end{array}$ & $\begin{array}{l}20.61 \\
(12.62)\end{array}$ & $\begin{array}{l}2.86 \\
(0.55)\end{array}$ \\
\hline & Chair & $\begin{array}{l}18.34 \\
(10.55)\end{array}$ & $\begin{array}{l}31.52 \\
(25.29)\end{array}$ & $\begin{array}{l}22.78 \\
(17.22)\end{array}$ & $\begin{array}{l}2.82 \\
(0.82)\end{array}$ \\
\hline & Avatar & $\begin{array}{l}21.51 \\
(11.44)\end{array}$ & $\begin{array}{l}28.77 \\
(18.59)\end{array}$ & $\begin{array}{l}23.87 \\
(15.78)\end{array}$ & $\begin{array}{l}2.89 \\
(0.71)\end{array}$ \\
\hline
\end{tabular}

Correlations Correlational analysis of the log-transformed data revealed several significant correlations between the transformed angular error scores for the VR and paper SOTs (see Table 2). Overall, these measures were significantly positively correlated $(r=.47, p<.001)$. In the control condition $(r=.65$, $p<.001)$, performance on the VR and paper versions was highly correlated, and in the arrow condition $(r=.43, p=$ $.005)$ and the avatar condition $(r=.44, p=.004)$ performance on the VR and paper versions of the SOT were moderately positively correlated. However, comparison of the correlations using Fisher's r-to-z transformations indicated that the differences between these correlations were not significant (all zvalues below critical value).

Survey Strategies reported on the questionnaire (see Appendix) were categorized as either mental simulation or abstract as follows: if the participant selected either answer c or answer $\mathrm{d}$, which mentioned imagining themselves inside

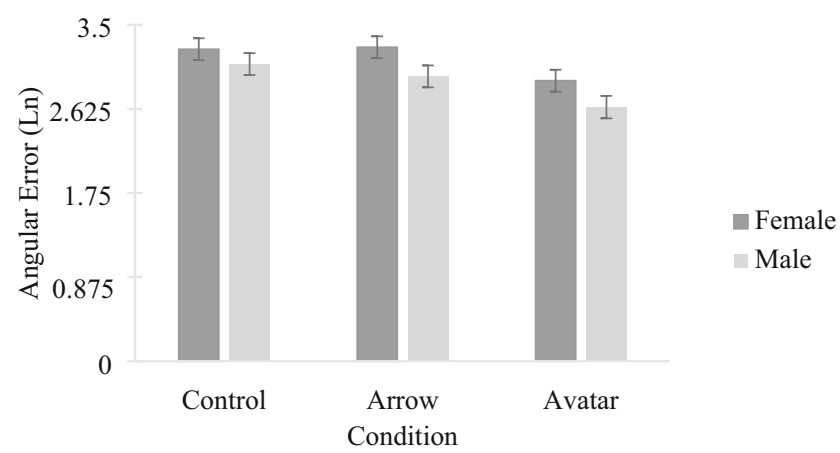

Fig. 3 Results from Experiment 1 showing mean Ln transformed angular error of males and females in the control, arrow, and avatar virtual reality conditions, with standard error bars $(n=120)$
Table 2. Correlations for Experiments 1 and 2 between Ln transformed mean angular errors on the virtual reality (VR) Spatial Orientation Test (SOT) and Ln transformed mean angular errors on the paper SOT

\begin{tabular}{lll}
\hline & & Paper SOT \\
\hline Experiment 1 & VR SOT Overall & $.47 * * *$ \\
& VR SOT Control & $.70^{* * *}$ \\
& VR SOT Arrow & $.44 * * *$ \\
& VR SOT Avatar & $.34 *$ \\
Experiment 2 & VR SOT Overall & $.47 * * *$ \\
& VR SOT Control & $.34 * *$ \\
& VR SOT Chair & $.60^{* * *}$ \\
& VR SOT Avatar & $.47 * * *$ \\
\hline
\end{tabular}

Note. Lower scores of angular error indicate better performance on the SOTs

$* p<.05, * * p<.01, * * * p<.001$

the array, their response was coded as "mental simulation." If participants chose either strategy a or strategy $b$, which mentioned imagining superimposing something else on the array to estimate the angle between the objects, their response was coded as "abstract." Text entry responses $(n=6)$ were coded by two independent coders who had complete agreement on whether they aligned with mental simulation or abstract strategies. Consistent with previous research using open-ended responses (Kozhevnikov \& Hegarty, 2001), almost all participants reported using a mental simulation strategy to complete both the VR SOT (105 mental simulation: 37 control, 35 arrow, 33 avatar; 15 abstract: three control, five arrow, seven avatar) and the paper SOT (111 mental simulation, nine abstract). A chi-square analysis indicated that there was no significant difference between strategies across conditions for the VR SOT, $\chi^{2}(2)=1.83, p=.40$, and an independent samples ttest indicated there was no significant difference in mean accuracy on the VR SOT between the mental simulation ( $M=$ $2.56, S D=.48)$ or abstract $(M=2.53, S D=.69)$ strategy groups $t(118)=-.24, p=.81$. There was also no significant effect of sex on strategy used on performance for either the VR SOT, $\chi^{2}(1)=.58, p=.45$ or the paper SOT, $\chi^{2}(1)=.37, p$ $=.55$.

One question on the survey asked participants in the avatar VR condition $(n=40)$ to rate how much they identified with the avatar on a five-point scale from 1 (not at all) to 5 (all of the time). The mean identification scores for males $(M=3.05$, $S D=1.10)$ and females $(M=2.35, S D=1.18)$ were not significantly different, $t(38)=-1.68, p=.10$.

\section{Discussion}

Findings from Experiment 1 indicate that performance was best in the avatar condition and performance in the arrow condition was no better than in the control condition. These 
results suggest that directionality alone is not sufficient to facilitate performance on this task, and that either the agency hypothesis or another mechanism is at play. They also suggest that the advantage of including a person in the display in previous research (Clements-Stephens et al., 2013, Tarampi et al., 2016) was not just because it reduced working memory load, as working memory load was also reduced in the arrow condition. A majority of participants in all conditions reported using a mental simulation strategy. The results suggest that the presence of an agent in the display did not affect which strategy people used, but rather affected how successfully people used a common mental simulation strategy. Although it was expected based on previous research (Tarampi et al., 2016) that the avatar condition would improve only female performance, both men and women performed better in this condition than in either the arrow or the control conditions.

Experiment 1 suggested that a directional cue alone (i.e., an arrow) was not sufficient for enhancing perspective-taking performance. A concern about the use of an arrow as a cue is that although it is a clear directional cue, we do not have much experience taking the perspective of arrows in everyday life. Moreover, there is ambiguity about where you position yourself in relation to an arrow (in front, behind, etc.). Experiment 2 resolves this ambiguity by utilizing a chair as the non-agentive directional cue. In addition to specifying both a location and a direction, a chair is a physical object that we interact with on a daily basis, and thus people have everyday experience to draw on in taking the perspective defined by a chair. In previous research, Kessler and Thomson (2010) explored the effects of a chair versus an avatar, in the context of how body position congruency influences performance on a perspective-taking task. Both an avatar and a chair facilitated performance on their task. Participants performed better when their body position was consistent with that of the avatar or the empty chair, particularly for larger angles of disparity between the participant and to-be-imagined position. Kessler and Thomson suggested that the avatar was not necessary to induce the mental self-rotation that occurs during spatial perspective taking. In Experiment 2, we directly compared an avatar condition to a chair condition in a VR display. In contrast with an arrow, which is a symbolic representation of a direction, a chair may facilitate mental simulation in that one can imagine oneself sitting in a chair, as chairs are concrete objects and most individuals have extensive, daily interactions chairs. An avatar and a chair both give a clear directional cue; both provide opportunity for enriched mental simulation or interaction, but differ in degree of agency.

Another limitation of the present experiment was that the instructions for the VR SOT did not include a sample item and $11 \%$ of participants were excluded for chance performance. To address this, we included a practice trial with feedback in Experiment 2. Moreover, the wording used in the avatar condition was different to that used by
Tarampi et al. (2016). Where Experiment 1 used the wording, "Imagine you are standing at the person, facing..." Tarampi et al.'s instructions were, "Take the perspective of the person, facing..." This difference in wording may be a possible explanation of why we did not fully replicate their results. To examine this possibility, we changed the wording in the avatar condition to be consistent with that used by Tarampi et al. (2016).

\section{Experiment 2}

In Experiment 2 we examined whether a directional cue (a chair) that is not an agent but is an object that can facilitate mental simulation or allows for interaction (such as imagining sitting in it) might give an advantage in perspective taking that is equivalent to the positive effects of including a human figure in the array found in Experiment 1. If agency is the critical factor that facilitates performance on this task due to automatic social sense making, then the avatar condition should produce better performance than either the chair or the control conditions. Alternatively, if a directional cue that is concrete and interactive is what facilitates performance, then performance in the avatar and chair conditions should be better than the control condition.

\section{Method}

Participants One hundred and ninety-one students from the University of California, Santa Barbara participated in this experiment for course credit. As in Experiment 1, we excluded participants who scored at or greater than chance levels of error $\left(4.7 \%^{5}\right)$ : three (female) from the control condition and three (female) from the avatar condition. Therefore, our final sample had 185 participants, age 17$30(M=19.10 S D=1.70)$ years. Again, a priori power analyses indicated that to detect a medium effect $\left(\eta_{p}{ }^{2}=\right.$ .13) of condition on VR SOT performance at a power of .95 , the necessary sample size would be $n=111$. The present study exceeds this requirement, and therefore is adequately powered to detect this effect.

Design This study had a two-factor between-subjects design. The independent variables were array condition (avatar, chair, and control), and sex, with 92 females (31 avatar, 31 chair, 30 control) and 93 males (31 avatar, 31 chair, 31 control) in the sample. As in Experiment 1, the dependent variable was average angular error across trials.

\footnotetext{
${ }^{5}$ Fewer participants are excluded here compared to Experiment $1(11 \%)$, suggesting that clarification of instructions and including a sample item improved task comprehension. Analyses including all participants do not change direction or significance of any effects.
} 
Materials The materials for this experiment were the same as in Experiment 1, except for the following changes: in the directional cue condition the arrow in the task array was replaced with a chair (see Fig. 4a), and the wording of the instructions for the avatar condition (see Fig. 4b) was changed from "Imagine standing at the person, facing $\underline{X}$, point to $\underline{Y}$," to read, "Take the perspective of the person, facing $\underline{X}$, point to $\underline{Y}$." The images provided in the survey questions regarding strategy used during the task were altered to reflect this change. Additionally, feedback was given to participants on the first practice trial, in the form of a line displaying the correct answer for the trial.

Procedure The procedure of this experiment was the same as in Experiment 1, with one change. Before the task practice trials, participants were told that on the next screen they would see an example trial with the correct answer drawn in. After advancing to the first practice trial, participants were instructed to move their arrow to indicate their answer, but to wait to submit their response. After the participants had moved their arrow, the experimenter reiterated that the line shown on the circle was the correct answer. Participants were asked to confirm that they understood why this was the right answer before proceeding to the experimental trials.

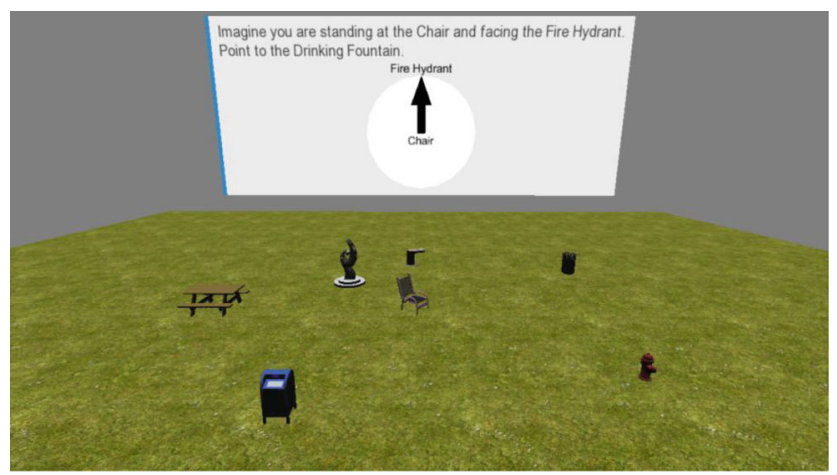

a Chair condition.

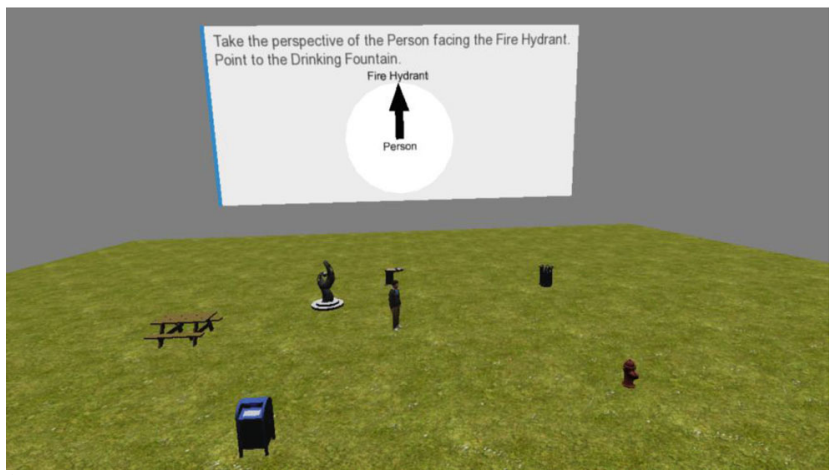

b Avatar condition.

Fig. 4 Screenshots of the virtual reality Spatial Orientation Test from the chair (a) and avatar (b) conditions in Experiment 2

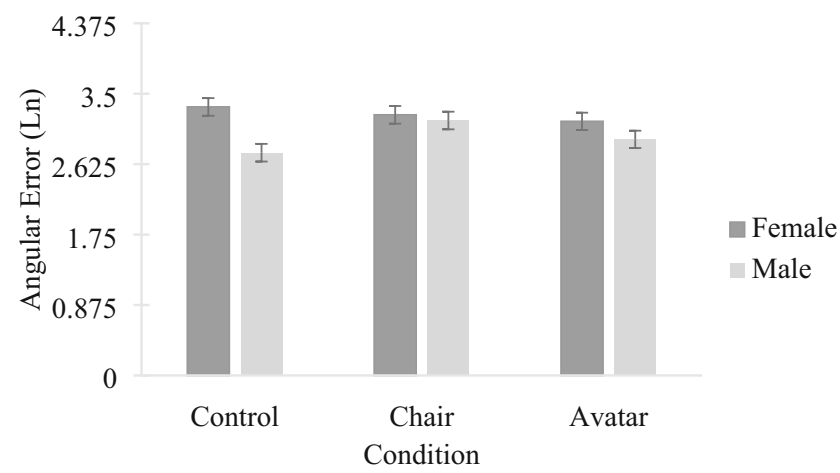

Fig. 5 Results from Experiment 2 showing mean Ln transformed angular error of males and females in the virtual reality Spatial Orientation Test with standard error bars $(n=185)$

\section{Results}

A small number of trials were not completed ${ }^{6}$ for the VR SOT $(M=.58, S D=1.34)$ and for the paper SOT $(M=1.15, S D=$ 1.79) and were assigned $90^{\circ}$ (chance performance). As in Experiment 1, the data were positively skewed so the data were natural-log transformed and all subsequent analyses reported used only log-transformed data; however, the direction and significance of the results did not differ if they were based on raw data, as shown in Table 1.

Virtual-reality Spatial Orientation Test If the agency of the avatar is the critical factor in facilitating performance, participants should perform significantly better in the avatar condition than in either the chair or the control conditions. Alternatively, if a directional cue that allows for everyday interaction is the critical factor that improves performance, the directional cue offered by either the chair or the avatar should boost performance. A 3 (condition: control, chair, avatar) $\times 2$ (sex: female, male) between-subjects ANOVA found a significant main effect of directional cue condition, $F(2,179)$ $=3.83, p=.02, \eta_{p}{ }^{2}=.04$. Post hoc (LSD) pairwise comparisons revealed that participants performed with lower angular error in the chair $(M=2.54, S D=.67, p=.008)$ and the avatar $(M=2.62, S D=.59, p=.05)$ conditions than in the control condition $(M=2.83, S D=.58)$ (see Fig. 5). Bayes Factors for these comparisons were 4.45 and 1.46 , respectively, indicating that the alternative hypotheses were more likely than the null hypothesis. There was no significant difference between the chair and the avatar conditions ( $p=.45$ ), and the Bayes Factor $\left(\mathrm{BF}_{10}\right)$ for this comparison was .25 , indicating that the data are roughly four times more likely to be observed under the null hypothesis than under the alternative hypothesis. These results

\footnotetext{
${ }^{6}$ Analyses of the average error for completed trials only indicated that the main effect of condition was marginal $(p=.054)$, with the same pattern as analyses conducted on average error with $90^{\circ}$ replacement for incomplete trials.
} 
support the mental simulation directional cue hypothesis rather than the agency (social sense-making) hypothesis.

There was also a significant main effect of sex, $F(1,179)=$ $15.78, p<.001, \eta_{p}{ }^{2}=.08$, such that males had significantly lower error on average $(M=2.49, S D=.56)$ than females $(M=$ $2.83, S D=.63$ ) across conditions, $d=.57$. There was no significant interaction between condition and sex, $p=.54$. Analysis of response-time data revealed no significant effect of condition $F(2,143)=1.16, p=.32$ or $\operatorname{sex} F(1,144)=3.26, p$ $=.07$, and no significant interaction, $p=.99$. For a complete list of means by sex and condition, see Table 1 .

Paper Spatial Orientation Test A between-subjects 2 (sex: female, male) $\times 3$ (condition: control, chair, avatar) ANOVA found a significant main effect of sex, $F(1,179)=16.53, p<.001, \eta_{p}{ }^{2}=$ .09 , such that males completed the task with significantly lower angular error on average $(M=2.50, S D=.58)$ than females $(M=$ 2.88, $S D=.68$ ) across conditions, $d=.60$ (see Table 1). As expected, there was no main effect of condition $F(2,179)=$ $.17, p=.85$, and no interaction of sex and condition, $p=.769$. This replicates the findings of Experiment 1.

Correlations Correlational analysis of the log-transformed data revealed several significant correlations between the transformed angular error scores for the VR and paper SOTs (see Table 2). Overall, these measures were significantly positively correlated $(r=.54, p<.001)$. In the control condition $(r=.45$, $p<.001)$, performance on the VR and paper versions was highly correlated, and in the chair condition $(r=.60, p<$ $.001)$ and the avatar condition $(r=.58, p<.001)$ performance on the VR and paper versions of the SOT was moderately positively correlated. As in Experiment 1, comparisons of these correlations using Fisher's r-to-z transformation indicated that they were not significantly different (all z-values below critical value). Moreover, a cross-experiment comparison indicated that there was no significant difference in correlation values for the control conditions $(z=1.37, p=.08)$ or the avatar conditions $(z=.89, p=.187)$ across experiments.

Survey Due to experimenter error, survey data were not collected for 13 subjects, so the following analyses were conducted with a smaller sample, $n=172$. As in Experiment 1, participant responses were categorized as consistent with either a mental simulation or abstract strategy (and two reviewers agreed on the classification of $n=6$ open-ended responses). As in Experiment 1, the majority of participants reported using a mental simulation strategy to complete both the paper SOT (155 mental simulation: 53 control, 54 chair, 48 avatar; 17 abstract: seven control, five chair, five avatar) and VR SOT (155 mental simulation, 17 abstract). A chi-square analysis indicated that there was no significant difference in strategy reported in the different cue conditions for either the VR SOT, $\chi^{2}(2)=.36, p=.84$, or the paper SOT, $\chi^{2}(2)=1.30, p=.52$.
Additionally, there was no significant effect of sex on strategy used for the VR SOT, $\chi^{2}(1)=3.38, p=.07$, or for the paper SOT, $\chi^{2}(1)=1.76, p=.18$. An independent samples t-test revealed that mean error on the VR SOT did not differ significantly between participants reporting a mental simulation $(M$ $=2.64, S D=.62)$ versus an abstract $(M=2.80, S D=.56)$ strategy $t(170)=1.07, p=.29$.

As in Experiment 1, another question on the survey revealed that there was no significant difference in identification with the avatar between men $(M=2.36, S D=1.04)$ and women $(M=2.58, S D=1.09), t(56)=.76, p=.45$.

\section{Discussion}

In Experiment 2 participants performed better in the chair and avatar conditions than in the control condition. Importantly, the chair and avatar conditions were not significantly different from each other and the dominant strategy in all conditions was to imagine oneself in the array (mental simulation). These results suggest that a directional cue that is a physical object with which we frequently interact is sufficient to facilitate mental simulation. A similar explanation could be applied to the avatar condition, suggesting that it is the ease of mentally simulating the position of the avatar, rather than its agentive nature (triggering a social sense-making process), which both facilitates the use of a mental simulation strategy and improves performance on this task.

\section{General discussion}

The main goal of this study was to explore why people perform better in a perspective taking task (the SOT) when the perspective to be imagined is that of a human figure in the task array (e.g., Shelton et al., 2012; Tarampi et al., 2016). In Experiment 1 we compared arrays with an avatar and an arrow to a control condition, and found that participants performed better in the avatar condition than in either the arrow or the control conditions. In Experiment 2, we compared arrays with an avatar and a chair to the control condition and found that participants performed better in the chair and avatar conditions than in the control condition. As shown in Table 3, an avatar, a chair, and an arrow differ in three aspects: agency, interactivity (in the sense that we are familiar with interacting with the cue),

Table 3. Aspects of the cues in the different experimental conditions of Experiments 1 and 2, and whether or not these cues facilitated performance relative to the control condition

\begin{tabular}{lllll}
\hline & Avatar & Chair & Arrow & \\
\hline Agency & Yes & No & No & Sufficient \\
Interactivity & Yes & Yes & No & Sufficient \\
Directionality & Yes & Yes & Yes & Insufficient \\
\hline
\end{tabular}


and directionality. The avatar has all three aspects, the chair has directionality and interactivity, and the arrow only has directionality. Our results suggest that while directionality alone is not sufficient to enhance performance on this task, a directional cue that is a familiar object with which we frequently interact is sufficient to improve performance. Contrary to the social mechanism hypothesis, an agentive cue is not necessary to improve perspective-taking performance.

In both experiments the avatar condition was completed with significantly less error than the control condition. This aspect of the study replicates Tarampi et al. (2016) and is also consistent with the results of Clements-Stephens et al. (2013) and Tversky and Hard (2009), demonstrating improved performance when the to-be-imagined heading was specified by an agent. Tarampi et al. suggest that this effect is the result of engagement of either mental simulation or social cognitive systems. The present results indicate that the interactivity factor is sufficient for facilitating perspective taking and there is no additional facilitation by social stimuli.

Further, in both experiments and for arrays with different directional cues, a majority of participants reported a mental simulation strategy for performing the task. These results suggest that directional cues do not alter the strategies adopted for this task. Rather they appear to affect how easily participants can employ mental simulation. In this context, mental simulation involves simulating a bodily transformation in order to complete the task, consistent with ideas of mental simulation from grounded and embodied cognition literature (e.g., Kessler \& Rutherford, 2010). It should be acknowledged that the strategy questionnaire was multiple-choice format, and therefore could be viewed as guiding participants to categorize their strategies in a particular way. However, our results are consistent with open-ended strategy descriptions in previous research on this task (Kozhevnikov \& Hegarty, 2001).

Our results suggest that although an arrow is familiar as a symbol that is used to indicate direction, it is difficult to mentally simulate taking the perspective of an arrow. Specifically, a mental simulation strategy in the arrow condition could have entailed imagining that the participant was the arrow, or that they were standing in front of or behind the arrow, so that their location in the display was ambiguous. In contrast, the chair offered a more concrete directional cue that participants are also used to interacting with. One possible mental simulation strategy used in the chair condition might be to imagine sitting in the chair, which specifies a single location in the array. In future research, open-ended strategy reports should be used to gain more insight into the specific nature of people's mental simulations in this task.

In the experiments conducted by Shelton et al. (2012) and Clements-Stephens et al. (2013), the critical factor in the high and medium agency conditions might also have been that they facilitated mental simulation. Both the artist model and Barbie $^{\mathrm{TM}}$ doll used in their research might have been anthropomorphized and seen as agents. A block figure attributed with conceptual agency ("alien") or a block figure with eyes may also facilitate mental simulation by seeming more human and easier to anthropomorphize. Interestingly, Shelton et al. (2012) found no difference in performance between an artist model and a camera. One can easily imagine interacting with a camera and this might facilitate mental simulation for perspective taking, as it did in the chair condition in Experiment 2 and in the study by Kessler and Thomson (2010), by establishing a clear position relative to the objects in the display, which in turn enables you to imagine yourself in the array. Finally, Shelton et al. also found no difference between these conditions and a "triangle" condition (a triangular block on a pedestal). While this condition is similar to our arrow, a difference is that the cue in their experiment was a physical three-dimensional object.

The motivations for translating the SOT into a VR format were to develop a more naturalistic measure of perspective taking in a scene with context, as is used in the developmental literature (e.g., Epley, Morewedge, \& Keysar, 2004; Huttenlocher \& Presson, 1973, 1979; Newcombe \& Frick, 2010; Newcombe, 1989; Surtees, Apperly, \& Samson, 2013), in contrast to the original paper SOT, which shows a more abstract top-down view that could have relied in part on map reading ability. In the VR SOT the plane of the array and the plane of the answer circle were not aligned. The primary reason for this was to maintain consistency with the answer circle used in the original SOT, even though this may increase the difficulty of the VR task overall. Despite this difference, the paper and VR versions of the task were moderately correlated in both experiments, suggesting that they measure the same ability.

Although the VR SOT had a more realistic and immersive display compared to the map-like display of the paper SOT, the VR versions tended to be more difficult and have higher error (see Table 1). This may be due to the difference in viewing angle of the array objects and the answer circle in the VR task. The answer circle was presented upright in the picture plane but represented a birds' eye view, while the array was viewed from an oblique $125^{\circ}$ angle. Imagining the angle between objects in the array from one viewpoint and then translating that to the aerial view of the answer circle may have added difficulty to the VR SOT, in contrast with the paper SOT in which both the array and answer circle are viewed from the same (top-down) viewing angle. Given that the VR and paper SOTs were intentionally presented in a fixed order, better performance in the paper SOTs might also reflect a practice effect.

Taken together, these experiments demonstrate sex differences in the SOT, both in the original paper and the new VR format. However, the present study did not replicate the interaction of condition and sex observed by Tarampi et al. (2016). Specifically, in this study both men and women had better performance when the directional cue was an avatar. This may be because men in the Tarampi et al. study performed close to ceiling in the control version of the paper task, which depicted 
both the array and the answer circle from the same viewing angle. In contrast, the more difficult VR task, which involved a change of viewing angle to relate the view of the array to the view of the answer circle, may have been more challenging for men, so that there was more room for improvement in their performance due to the avatar and chair directional cues.

More research is necessary to examine the multitude of factors that could influence performance on perspective-taking tasks. Future research should examine other non-human directional cues, particularly those that we interact with (e.g., cameras, cars) and those that we do not interact with (e.g., weather veins, flags). This research should include different frames of reference in conjunction with potential for mental simulation; for instance, does the conceptual "front" of the person align with the conceptual front of the object faced. Future studies should also consider ambiguously or incorrectly oriented human figures to further isolate agentive versus directional components that may influence performance. For example, the array could include a human figure that always faces forward, therefore not facing any of the objects and not changing its facing direction between trials.

The present experiments provide insights about the cognitive processes that underlie perspective-taking ability, particularly in environments that contain social and directional cues. Our research suggests that people perform perspective-taking tasks primarily using a mental simulation strategy in which they imagine themselves in the array. Directional cues that facilitate this mental simulation may support enhanced performance, regardless of whether they are agents or objects with which we can imagine interacting. Given large individual differences in perspective taking, future research should build upon these insights to guide the development of training strategies for perspective taking and other spatial abilities.

Author Note This research was supported by a grant to M.H. from the Academic Senate of the University of California, Santa Barbara. We would like to thank David Sañosa for his extensive work in developing the VR SOT program. We would also like to acknowledge Alexander Boone, Sarah Creem-Regehr, Alinda Friedman, Carol He, and Margaret Tarampi for their thoughtful comments on earlier versions of this manuscript.

\section{Appendix}

Survey Strategy Questions - for both paper and virtual reality (VR) Spatial Orientation Tests (SOTs)

a) Other students have reported a range of strategies that they used to do these tasks. Please indicate which strategy is closest to the one that you used when doing each of the tasks. For this task what strategy did you use the most: a) I mentally superimposed the whole array of objects on the answer circle to formulate my answer.

b) I superimposed the answer circle on the array of objects to formulate my answer.

c) I imagined drawing the angle between the object I was facing, my imagined location, and the target object. I then moved and rotated that angle and superimposed on the answer circle to formulate my answer.

d) I imagined myself being in the array, at the location I was told to imagine, turning my body to the imagined facing direction, and figured out where the target object would be in relation to my body.

e) I did not use any of these strategies. I used the following strategy:

Note: The strategy questions were scored as follows: strategy choices (a) and (b) were coded as abstract, while choices (c) and (d) were coded as mental simulation. Any text entries were coded by two independent raters as either abstract or mental simulation.

Publisher's note Springer Nature remains neutral with regard to jurisdictional claims in published maps and institutional affiliations.

\section{References}

Allen, G. L., K. C. Kirasic, S. H. Dobson, R. G. Long, \& S. Beck (1996). Predicting environmental learning from spatial abilities: An indirect route, Intelligence, 22, 327-355.

Baron-Cohen, S., Wheelwright, S., Skinner, R., Martin, J., \& Clubley, E. (2001). The autism-spectrum quotient (AQ): Evidence from Asperger syndrome/high-functioning autism, males and females, scientists and mathematicians. Journal of Autism and Developmental Disorders, $31(1), 5-17$.

Barsalou, L. W. (2008). Grounded cognition. Annual Review of Psychology, 59, 617-645.

Clements-Stephens, A. M., Vasiljevic, K., Murray, A. J., \& Shelton, A. L. (2013). The role of potential agents in making spatial perspective taking social. Frontiers in Human Neuroscience, 7, 497.

Clinton, J. A., Magliano, J. P., \& Skowronski, J. J. (2018). Gaining perspective on spatial perspective taking. Journal of Cognitive Psychology, 30(1), 85-97.

Costantini, M., Ambrosini, E., Cardellicchio, P., \& Sinigaglia, C. (2013). How your hand drives my eyes. Social cognitive and affective neuroscience, 9(5), 705-711.

De Freitas, E., \& Sinclair, N. (2012). Diagram, gesture, agency: Theorizing embodiment in the mathematics classroom. Educational Studies in Mathematics, 80(1-2), 133-152.

Epley, N., Morewedge, C. K., \& Keysar, B. (2004). Perspective taking in children and adults: Equivalent egocentrism but differential correction. Journal of Experimental Social Psychology, 40(6), 760-768.

Fields, A. W. \& A. L. Shelton (2006). Individual skill differences and large-scale environmental learning, Journal of Experimental Psychology: Learning, Memory, and Cognition, 32, 506-515.

Flavell, J. H., Everett, B. A., Croft, K., \& Flavell, E. R. (1981). Young children's knowledge about visual perception: Further evidence for the Level 1-Level 2 distinction. Developmental Psychology, 17(1), 99. 
Galinsky, A. D., Ku, G., \& Wang, C. S. (2005). Perspective-taking and self-other overlap: Fostering social bonds and facilitating social coordination. Group Processes \& Intergroup Relations, 8(2), 109-124.

Gardner, M., Brazier, M., Edmonds, C. J., \& Gronholm, P. (2013). Strategy modulates spatial perspective-taking: evidence for dissociable disembodied and embodied routes. Frontiers in human neuroscience, 7, 457.

Hegarty, M. (1992). Mental animation: Inferring motion from static displays of mechanical systems. Journal of Experimental Psychology: Learning, Memory, and Cognition, 18(5), 1084.

Hegarty, M. (2004). Mechanical reasoning by mental simulation. Trends in cognitive sciences, $8(6), 280-285$.

Hegarty, M., \& Waller, D. (2004). A dissociation between mental rotation and perspective-taking spatial abilities. Intelligence, 32(2), 175-191.

Huttenlocher, J., \& Presson, C. C. (1973). Mental rotation and the perspective problem. Cognitive Psychology, 4(2), 277-299.

Huttenlocher, J., \& Presson, C. C. (1979). The coding and transformation of spatial information. Cognitive Psychology, 11(3), 375-394.

JASP Team. T.(2017). JASP (Version 0.8. 1.2)[Computer software].

Kessler, K., \& Thomson, L. A. (2010). The embodied nature of spatial perspective taking: embodied transformation versus sensorimotor interference. Cognition, 114(1), 72-88.

Kozhevnikov, M., \& Hegarty, M. (2001). A dissociation between object manipulation spatial ability and spatial orientation ability. Memory \& Cognition, 29(5), 745-756.

Kozhevnikov, M., Motes, M. A., Rasch, B. \& Blajenkova, O. (2006), Perspective-taking vs. mental rotation transformations and how they predict spatial navigation performance, Applied Cognitive Psychology, 20, 397-417.

May, M. (2004). Imaginal perspective switches in remembered environments: Transformation versus interference accounts. Cognitive Psychology, 48(2), 163-206.

Money, J., Alexander, D., \& Walker, H. T. (1965). A standardized roadmap test of direction sense: Manual. Johns Hopkins Press.

Newcombe, N. (1989). The development of spatial perspective taking. In Advances in Child Development and Behavior (Vol. 22, pp. 203247). JAI.

Newcombe, N. S., \& Frick, A. (2010). Early education for spatial intelligence: Why, what, and how. Mind, Brain, and Education, 4(3), 102-111.

Niedenthal, P. M., Barsalou, L. W., Winkielman, P., Krauth-Gruber, S., \& Ric, F. (2005). Embodiment in attitudes, social perception, and emotion. Personality and social psychology review, 9(3), 184-211.
Núñez, R. E., Edwards, L. D., \& Matos, J. F. (1999). Embodied cognition as grounding for situatedness and context in mathematics education. Educational studies in mathematics, 39(1-3), 45-65.

Piaget, J., \& Inhelder, B. (1967). The coordination of perspectives. The Child's Conception of Space, 8, 209-246.

Presson, C. C., \& Montello, D. R. (1994). Updating after rotational and translational body movements: Coordinate structure of perspective space. Perception, 23(12), 1447-1455.

Rieser, J. J. (1989). Access to knowledge of spatial structure at novel points of observation. Journal of Experimental Psychology: Learning, Memory, and Cognition, 15(6), 1157.

Samson, D., Apperly, I. A., Braithwaite, J. J., Andrews, B. J., \& Bodley Scott, S. E. (2010). Seeing it their way: evidence for rapid and involuntary computation of what other people see. Journal of Experimental Psychology: Human Perception and Performance, 36(5), 1255.

Schinazi, V. R., Nardi, D., Newcombe, N. S., Shipley T. F., \& Epstein, R. (2013), Hippocampal size predicts rapid learning of a cognitive map in humans, Hippocampus, 23 (6), 515-528

Sebanz, N., Knoblich, G., \& Prinz, W. (2003). Representing others' actions: just like one's own? Cognition, 88(3), B11-B21.

Shelton, A. L., Clements-Stephens, A. M., Lam, W. Y., Pak, D. M., \& Murray, A. J. (2012). Should social savvy equal good spatial skills? The interaction of social skills with spatial perspective taking. Journal of Experimental Psychology: General, 141(2), 199.

Sholl, M. J., Acacio, J. C., Makar, R. O., \& Leon, C. (2000). The relation of sex and sense of direction to spatial orientation in an unfamiliar environment. Journal of Environmental Psychology, 20(1), 17-28.

Surtees, A. D. R., Apperly, I. A., \& Samson, D. (2013). The use of embodied self-rotation for visual and spatial perspective-taking. Frontiers in Human Neuroscience, 7, 698.

Tarampi, M. R., Heydari, N., \& Hegarty, M. (2016). A tale of two types of perspective taking: Sex differences in spatial ability. Psychological Science, 27(11), 1507-1516.

Tversky, B., \& Hard, B. M. (2009). Embodied and disembodied cognition: Spatial perspective-taking. Cognition, 110(1), 124-129.

Weisberg, S. M., Schinazi, V. R. Newcombe, N. S., Shipley, T. F, \& Epstein, R. A. (2014), Variations in cognitive maps: Understanding individual differences in navigation, Journal of Experimental Psychology: Learning, Memory, and Cognition, 40 (3), 669 - 682.

Zwickel, J. (2009). Agency attribution and visuospatial perspective taking. Psychonomic Bulletin \& Review, 16(6), 1089-1093. 